\title{
REGULAR TREE LANGUAGE RECOGNITION WITH STATIC INFORMATION
}

\author{
Alain Frisch \\ École Normale Supérieure \\ Alain.Frisch@ens.fr
}

\begin{abstract}
This paper presents our compilation strategy to produce efficient code for pattern matching in the CDuce compiler, taking into account static information provided by the type system.
\end{abstract}

\section{Introduction}

Emergence of XML[BPSM98] has given tree automata theory a renewed importance[Nev02]. Indeed, XML schema languages such as DTD, XMLSchema[TBMM01, SW03], Relax-NG describe more or less regular languages of XML documents (considered as trees). Consequently, recent XML-oriented typed programming languages such as XDuce [Hos00, HP02], CDuce [BCF03, FCB02], Xtatic [GP03] have type algebras where types denote regular tree languages. An essential ingredient of these languages is a powerful pattern matching operation. A pattern is a declarative way to extract information from an XML tree. Because of this declarative nature, language implementors have to propose efficient execution models for pattern matching.

This paper describes our approach in implementing pattern matching in CDuce ${ }^{1}$. To simplify the presentation, the paper studies only a restricted form of pattern matching, without capture variables and with a very simple kind of trees. Of course, our implementation handles capture variables and the full set of types and patterns constructors in CDuce. In the simplified form, the pattern matching problem is a recognition problem, namely deciding whether a tree $v$ belongs to a regular tree language $X$ or not. If the regular language is given by a tree automaton, a top-down recognition algorithm may have to backtrack, and the recognition time is not linear in the size of the input tree. It is well-known that any tree automaton can be transformed into an equiva-

${ }^{1}$ CDuce is available for download at http: //www. cduce. org/ . 
lent bottom-up deterministic automaton, which ensures linear execution time. However, the size of the automaton may be huge even for simple languages, which can make this approach unfeasible in practice.

The static type system of the language provides an upper approximation for the type of the matched tree $v$, that is some regular language $X_{0}$ such that $v$ is necessarily in $X_{0}$. Taking this information into account, it should be possible to avoid looking at some subtree of $v$. However, classical bottom-up tree automata are bound to look at the whole tree, and they cannot take this kind of static knowledge into account. Let us give an example to illustrate this point. Consider the following CDuce program:

\section{type $A=\langle a\rangle[A *]$ \\ type $B=\langle b\rangle[B *]$}

\section{Let $f((A \mid B) \rightarrow$ Int $) A \quad \rightarrow 0 \mid B \rightarrow 1$ \\ let $g((A \mid B)->$ Int $)\langle a\rangle_{-}->0 \mid \ldots$}

The first lines introduce two types A and B. They denote XML documents with only $\langle a\rangle($ resp. $<b>$ ) tags and nothing else. Then two functions $f$ and $g$ are defined. Both functions take an argument which is either a document of type A or of type B. They return 1 when the argument is of type A, and 0 when the argument is of type B. The declaration of $g$ suggests an efficient execution schema: one just has to look at the root tag to answer the question. Instead, if we consider only the body of $f$, we have to look at the whole argument, and check that every node of the argument is tagged with $<a>$ (resp. with $<\mathrm{b}\rangle$ ); whatever technique we use - deterministic bottom-up or backtracking top-down - it will be less efficient than $\mathrm{g}$.

But if we use the information given by the function interface, we know that the argument is necessarily of type $A$ or of type $B$, and we can compile $f$ exactly as we compile $g$. This example demonstrates that taking static information into account is crucial to provide efficient execution for declarative patterns as in $f$.

Contributions. The main contributions of this paper are the definition of a new kind of deterministic bottom-up tree automata, called NUA (non-uniform automata) and a compilation algorithm that produces an efficient NUA equivalent to a given non-deterministic (classical) automaton, taking into account static knowledge about the matched trees.

A central idea in XDuce-like languages is that XML documents live in an untyped world and that XML types are structural. This is in contrast with the XML Schema philosophy, whose data model (after validation) attaches type names to XML nodes. Moreover, in XML Schema, the context and the tag of an element are enough to know the exact XML Schema type of the element. In XDuce-like languages, in general, one may have to look deep 
inside the elements to check type constraints. Our work shows how an efficient compilation of pattern matching can avoid this costly checks: our compilation algorithm detects when the context and the tag are enough to decide of the type of an element without looking at its content. This work supports the claim that a structural data model $a$ la XDuce can be implemented as efficiently as a data model with explicit type names à la XML Schema.

Related work. Levin [Lev03] also addresses the implementation of pattern matching in XDuce-like programming languages. He introduces a general framework (intermediate language, matching automata) to reason about the compilation of patterns, and he proposes several compilation strategies. $\mathrm{He}$ leaves apart the issue of using static types for compilation, which is the main motivation for our work. So the two works are complementary: our compilation algorithm could probably be re-cast in his formalism.

Neumann and Seidl [NS98] introduce push-down automata to locate efficiently nodes in an XML tree. Our automata share with push-down automata the idea of threading a control-state through the tree. The formalisms are quite different because we work with simpler kind of automata (binary trees with labeled leaves, whereas they have unranked labeled forests), and we explicitly distinguish betwen control states (threaded through the tree) and results (used in particular to update the state). However, using an encoding of unranked trees in binary trees, we believe that the two notions of automata are isomorphic. But again, they don't address the issue of using static information to improve the automata, which is our main technical contribution. It should be possible to adapt our compilation algorithm to their push-down automata setting, but it would probably result in an extremely complex technical presentation. This motivates us working with simpler kinds of tree and automata.

\section{Technical framework}

In this section, we introduce our technical framework. We consider one of the simplest form of trees: binary trees with labeled leafs and unlabeled nodes. Any kind of ordered trees (n-ary, ranked, unranked; with or without labeled nodes) can be encoded, and the notion of regular language is invariant under these encodings. (Note that the encodings change the expressive power of top-down deterministic tree automata, but this is not the case for the "nonuniform" automata we are going to define.) Using this very simple kind of trees simplifies the presentation.

\subsection{Trees and classical tree automata}

DEFINITION 1 Let $\boldsymbol{\Sigma}$ be a (fixed) finite set of symbols. A tree $\boldsymbol{v}$ is either a symbol $\boldsymbol{a} \in \mathbf{\Sigma}$ or a pair oftrees $\left(v_{1}, v_{2}\right)$. The set of trees is written $\mathcal{V}$. 
DEFINITION 2 (TREE AUTOMATON) $A$ (non-deterministic) tree automaton (NDTA) is a pair $\mathcal{A}=(R, \delta)$ where $R$ is a finite set of nodes, and $\delta \subseteq(\Sigma \times$ $R) \cup(R \times R \times R)$.

Each node $r$ in a NDTA defines a subset $\mathcal{A} \llbracket r \rrbracket$ of $\mathcal{V}$. These sets can be defined by the following mutually recursive equations:

$$
\mathcal{A} \llbracket r \rrbracket=\{a \in \Sigma \mid(a, r) \in \delta\} \cup \bigcup_{\left(r_{1}, r_{2}, r\right) \in \delta} \mathcal{A} \llbracket r_{1} \rrbracket \times \mathcal{A} \llbracket r_{2} \rrbracket
$$

We write $\mathcal{A} \llbracket r \rrbracket^{2}=\mathcal{A} \llbracket r \rrbracket \cap(\mathcal{V} \times \mathcal{V})$. By definition, a regular language is a subset of the form $\mathcal{A} \llbracket r \rrbracket$ for some NDTA $\mathcal{A}$ and some node $r$. We say that this language is defined by $\mathcal{A}$. There are two classical notions of deterministic tree automata: (1) Top-down deterministic automata (TDDTA) satisfy the property: $\left\{\left(r_{1}, r_{2}\right) \mid\left(r_{1}, r_{2}, r\right) \in \delta\right\}$ has at most one element for any node $r$. These automata are strictly weaker than NDTA in terms of expressive power (they cannot define all the regular languages). (2) Bottom-up deterministic automata (DTA) satisfy the property: $\left\{r \mid\left(r_{1}, r_{2}, r\right) \in \delta\right\}$ has at most one element for any pair of nodes $\left(r_{1}, r_{2}\right)$, and similarly for the sets $\{r \mid(a, r) \in \delta\}$ with $a \in \Sigma$. These automata have the same expressive power as NDTA.

REMARK 3 We use a non-standard terminology of nodes instead of states. The reason is that we are going to split this notion in two: results and control states. Results will correspond to nodes in a DTA, and control states will correspond to nodes in TDDTA.

In order to motivate the use of a different kind of automaton, let us introduce different notions of context. During the traversal of a tree, an automaton computes and gathers information. The amount of extracted information can only depend on the context of the current location in the tree. A top-down recognizer (for TDDTA) can only propagate information downwards: the context of a location is thus the path from the root to the location ("upward context"). A bottom-up recognizer propagates information upwards: the context is the whole subtree rooted at the current location ("downward context").

Top-down algorithms are more efficient when the relevant information is located near the root. For instance, going back to the CDuce example in the introduction, we see easily that the function $g$ should be implemented by starting the traversal from the root of the tree, since looking only at the root tag is enough. Patterns in CDuce tend to look in priority near the root of the trees instead of their leafs. However, because of their lack of expressive power, pure TDDTA cannot be used in general. Also, since they perform independant computations of the left and the right children of a location in a tree, they cannot use information gathered in the left subtree to guide the computation in the right subtree. 
The idea behind push-down automata is to traverse each node twice. A location is first entered in a given context, some computation is performed on the subtree, and the location is entered again with a new context. When a location is first entered, the context is the path from the root, but also all the "left siblings" of these locations and their subtrees (we call this the "up/left context" of the location). After the computation on the children, the context also includes the subtree. The notion of non-uniform automata we are going to introduce is a slight variation on this idea: a location is entered three times. Indeed, when computing on a tree which is a pair, the automaton considers the left and right subtrees sequentially. Between the two, the location is entered again to update its context, and the automaton uses the information gathered on the left subtree to guide the computation on the right subtree. This richer notion of context allows to combine the advantages of DTA and TDDTA, and more.

\subsection{Non-uniform automata}

We now introduce a new kind of tree automaton: non-uniform automata (NUA in short). They can be seen as (a generalization of) a merger between DTA and TDDTA. Let us call "results" (resp. "control states") the nodes of DTA (resp. TDDTA). We are going to use these two notions in parallel. A current "control state" is threaded and updated during a depth-first left-to-right traversal of the tree (this control generalizes the one of TDDTA, where the state is only propagated downwards), and each control state $q$ has its own set of results $R(q)$. Of course, the transition relation depends on $q$.

When the automaton has to deal with a tree $\left(v_{1}, v_{2}\right)$ in a state $q$, it starts with some computation on $v_{1}$ using a new state $q_{1}=\operatorname{left}(q)$ computed from the current one, as for a TDDTA. This gives a result $\boldsymbol{r}_{1}$ which is immediately used to compute the state $q_{2}=\operatorname{right}\left(q, r_{1}\right)$. Note that contrary to TDDTA, $q_{2}$ depends not only on $q$, but also on the computation performed on the left subtree. The computation on $v_{2}$ is done from this state $q_{2}$, and it returns a result $r_{2}$. As for classical bottom-up deterministic automata, the result for $\left(v_{1}, v_{2}\right)$ is then computed from $r_{1}$ and $r_{2}$ (and $q$ ). Let us formalize the definition of non-uniform automata. We define only the deterministic version.

DEFINITION 4 A non-uniform automaton $\mathcal{A}$ is given by a finite set of states $Q$, and for each state $\boldsymbol{q} \in \mathbf{Q}$ :

- A finite set of results $R(q)$.

- A state $\operatorname{left}(q) \in Q$.

- For any result $r_{1} \in R(\operatorname{left}(q))$, a state $\operatorname{right}\left(q, r_{1}\right) \in Q$. 
- Foranyresult $r_{1} \in R(\operatorname{left}(q))$, and any result $r_{2} \in R\left(\operatorname{right}\left(q, r_{1}\right)\right)$, a result $\delta^{2}\left(q, r_{1}, r_{2}\right) \in R(q)$.

- A partialfunction $\delta^{0}(q,-): \Sigma \rightarrow R(q)$.

The result of the automaton from a state $q$ on an input $v \in \mathcal{V}$, written $\mathcal{A}(q, v)$, is the element of $R(q)$ defined by induction on $v$ :

$$
\begin{array}{ll}
\mathcal{A}(q, a) & =\delta^{0}(q, a) \\
\mathcal{A}\left(q,\left(v_{1}, v_{2}\right)\right) & =\delta^{2}\left(q, r_{1}, r_{2}\right) \text { where }
\end{array} \quad\left\{\begin{array}{l}
r_{1}=\mathcal{A}\left(\operatorname{left}(q), v_{1}\right) \\
r_{2}=\mathcal{A}\left(\operatorname{right}\left(q, r_{1}\right), v_{2}\right)
\end{array}\right.
$$

Because the functions $\delta^{0}(q,-)$ are partial, so are the $\mathcal{A}(q,-)$. We write $\operatorname{Dom}(q)$ for the set of trees $v$ such that $\mathcal{A}(q, v)$ is defined.

Our definition of NUAs (and more generally, the class of push down automata [NS98]) is flexible enough to simulate DTA and TDDTA (without explosion of size). Indeed, the definition of a NUA boils down to that of a DTA when $Q$ is a singleton $\{q\}$ : the set of results of the NUA (for the only state) corresponds to the set of nodes of the DTA. It is also possible to convert a TDDTA to a NUA of the same size: The set of states of the NUA corresponds to the set of nodes of the TDDTA, and all the states have a single result.

A pair $(q, r)$ with $q \in Q$ and $r \in R(q)$ is called a state-result pair. For such a pair, we write $\mathcal{A} \llbracket q ; r \rrbracket=\{v \mid \mathcal{A}(q, v)=r\}$ for the set of trees yielding result $\boldsymbol{r}$ starting from initial state $\boldsymbol{q}$. The reader is invited to check that a NUA can be interpreted as a non-deterministic tree automata whose nodes are stateresult pairs. Consequently, the expressive power of NUAs (that is the class of languages of the form $\mathcal{A} \llbracket q ; r \rrbracket)$ is the same as NDTAs (ie: they can define only regular languages). The point is that the definition of NUAs gives an efficient execution strategy.

Running a NUA. The definition of $\mathcal{A}(q, v)$ defines an efficient algorithm that operates in linear time with respect to the size of $v$. We will only run this algorithm for trees $v$ which are known a priori to be in $\operatorname{Dom}(q)$. This is because of the intended use of the theory (compilation of CDuce pattern matching): indeed, the static type system in CDuce ensures exhaustivity of pattern matching.

An important remark: the flexibility of having a different set of results for each state makes it possible to short-cut the inductive definition and completely ignore subtrees. Indeed, as soon as the algorithm reaches a subtree $\boldsymbol{v}^{\prime}$ in a state $q^{\prime}$ such that $R\left(q^{\prime}\right)$ is a singleton, it can directly return without even looking at $v^{\prime}$.

\section{The algorithm}

Different NUA can perform the same computation with different complexities (that is, they can ignore more or fewer subtrees of the input). To obtain 
efficient NUA, the objective is to keep the set of results $\boldsymbol{R}(\boldsymbol{q})$ as small as possible, because when $R(q)$ is a singleton, we can drop the corresponding subtree (and having $R(q)$ small will help "subsequent" $R\left(q^{\prime}\right)$ to be singletons).

Also, we want to build NUAs that take static information about the input trees into account. Hopefully, we have the opportunity of defining partial states, whose domain is not the whole set of trees.

In this section, we present an algorithm to build an efficient NUA to solve the dispatch problem under static knowledge.

Given a regular language $X_{0}$ (the input domain) and given regular languages $X_{1}, \ldots, X_{n}$ (the dispatch alternatives), we want to compute efficiently for any tree $v \in X_{0}$ the set $\left\{i \mid i \in\{1, \ldots, n\}, v \in X_{i}\right\}$.

\subsection{Intuitions}

Let us consider four regular languages $X_{1}, X_{2}, X_{3}, X_{4}$, and let $X=\left(X_{1} \times\right.$ $\left.X_{2}\right) \cup\left(X_{3} \times X_{4}\right)$. Imagine we want to recognize the language $X$ without static information $\left(X_{0}=\mathcal{V}\right)$. If we are given a tree $\left(v_{1}, v_{2}\right)$, we must first perform some computation on $\boldsymbol{v}_{1}$. Namely, it is enough to know, after this computation, if $v_{1}$ is in $X_{1}$ or not, and similarly for $\boldsymbol{X}_{\mathbf{3}}$. It is not necessary to do any other computation; for instance, we don't care whether $v_{1}$ is in $X_{2}$ or not. According to the presence of $v_{1}$ in $X_{1}$ and/or $X_{3}$, we continue with different computations of $v_{2}$ :

- It $v_{1}$ is neither in $X_{1}$ nor in $X_{3}$, we already know that $v$ is not in $X$ without looking at $\boldsymbol{v}_{2}$. We can stop the computation immediately.

- If $\boldsymbol{v}_{\mathbf{1}}$ is in $\boldsymbol{X}_{\mathbf{1}}$ but not in $X_{\mathbf{3}}$, we have to check whether $\boldsymbol{v}_{\mathbf{2}}$ is in $\boldsymbol{X}_{\mathbf{2}}$.

- If $v_{1}$ is in $X_{\mathbf{3}}$ but not in $X_{1}$, we have to check whether $v_{2}$ is in $X_{\mathbf{4}}$.

- If $\boldsymbol{v}_{1}$ is in $\boldsymbol{X}_{\mathbf{1}}$ and in $\boldsymbol{X}_{\mathbf{3}}$, we must check whether $\boldsymbol{v}_{\mathbf{2}}$ is in $\boldsymbol{X}_{\mathbf{2}}$ or not, and in $X_{4}$ or not. But actually, this is too much. We only have to find out whether it is in $X_{2} \cup X_{4}$ or not, and this can be easier to do (for instance, if $X_{2} \cup X_{4}=\mathcal{V}$, we don't have anything to do at all).

This is the general case, but in some special cases, it is not necessary to know both whether $v_{1}$ is in $X_{1}$ and whether it is in $X_{3}$. For instance, imagine that $X_{2}=X_{4}$. Then we don't have to distinguish the three cases $v_{1} \in X_{1} \backslash X_{3}$, $v_{1} \in X_{3} \backslash X_{1}, v_{1} \in X_{1} \cap X_{3}$. Indeed, we only need to check whether $v_{1}$ is in $X_{1} \cup X_{3}$ or not. We could as well have merged $X_{1} \times X_{2}$ and $X_{3} \times X_{4}$ into $\left(X_{1} \cup X_{3}\right) \times X_{2}$ in this case. We can alsomerge $X_{1} \times X_{2}$ and $X_{3} \times X_{4}$ if one of them is a subset of the other.

Now consider the case where $\boldsymbol{X}_{\mathbf{0}}$ is a proper subset of $V$ (non trivial static information). If for instance, $X_{0} \cap\left(X_{1} \times X_{2}\right)=\varnothing$, we can simply ignore the rectangle $X_{1} \times X_{2}$. Also, in general, we deduce some information about 
$v_{1}$ : it belongs to $\pi_{1}\left(X_{0}\right)=\left\{v_{1}^{0} \mid\left(v_{1}^{0}, v_{2}^{0}\right) \in X_{0}\right\}$. After performing some computation on $v_{1}$, we get more information. For instance, we may deduce $v_{1} \in X_{1} \backslash X_{3}$. Then we know that $v_{2}$ is in $\pi_{2}\left(X_{0} \cap\left(X_{1} \backslash X_{3}\right) \times \mathcal{V}\right)$. In general, we can combine the static information and the results we get for the a left subtree to get a better static information for the right subtree. Propagating a more precise information allows to ignore more rectangles.

The static information allows us to weaken the condition to merge two rectangles $X_{1} \times X_{2}$ and $X_{3} \times X_{4}$. Indeed, it is enough to check whether $\pi_{2}\left(X_{0} \cap\left(X_{1} \times X_{2}\right)\right)=\pi_{2}\left(X_{0} \cap\left(X_{3} \times X_{4}\right)\right)$ (which is strictly weaker than $\left.X_{2}=X_{4}\right)$.

In some cases, there are decisions to make. Imagine that $X_{0}=X_{1} \times$ $X_{2} \cup X_{3} \times X_{4}$, and we want to check if a tree $\left(v_{1}, v_{2}\right)$ is in $X_{1} \times X_{2}$. If we suppose that $X_{1} \cap X_{3}=\varnothing$ and $X_{2} \cap X_{4}=\varnothing$, we can work on $v_{1}$ to see if it is in $X_{1}$ or not, or we can work on $v_{2}$ to see if it is in $X_{2}$ or not. We don't need to do both, and we must thus choose which one to do. We always choose to perform some computation on $v_{1}$ if it allows to gain useful knowledge on $\boldsymbol{v}$. This choice allows to stop the top-down left-to-right traversal of the tree as soon as possible. This choice is relevant when considering the way CDuce encodes sequences and XML trees as binary trees. Indeed, the choice corresponds to: (1) extracting information from an XML tag to guide the computation on the content of the element, and (2) extracting information from the first children before considering the following ones.

\subsection{Types}

We have several regular languages $X_{0}, X_{1}, \ldots, X_{n}$ as inputs, and our algorithm produces other languages as intermediate steps. Instead of working with several different NDTA to define these languages, we assume that all the regular languages we will consider are defined by the same fixed NDTA $\mathcal{A}$ (each language is defined by a specific state of this NDTA). This assumption is not restrictive since it is always possible to take the (disjoint) union of several NDTA. Moreover, we assume that this NDTA has the following properties:

- Boolean-completeness. The class of languages defined by $\mathcal{A}$ (that is, the languages of the form $\mathcal{A} \llbracket r \rrbracket$ ), is closed under boolean operations (union, intersection, complement with respect to $\mathcal{V}$ ).

- Canonicity. If $\left(r_{1}, r_{2}, r\right) \in \delta$, then: $\mathcal{A} \llbracket r_{1} \rrbracket \neq \varnothing, \mathcal{A} \llbracket r_{2} \rrbracket \neq \varnothing$. Moreover, if we consider another pair $\left(r_{1}^{\prime}, r_{2}^{\prime}\right) \neq\left(r_{1}, r_{2}\right)$ such that $\left(r_{1}^{\prime}, r_{2}^{\prime}, r\right) \in \delta$, then $\mathcal{A} \llbracket r_{1} \rrbracket \cap \mathcal{A} \llbracket r_{1}^{\prime} \rrbracket=\varnothing$ and $\mathcal{A} \llbracket r_{2} \rrbracket \neq \mathcal{A} \llbracket r_{2}^{\prime} \rrbracket$.

It is well-known that the class of all regular tree languages is closed under boolean operations. The first property says that the class of languages defined by the fixed NDTA $\mathcal{A}$ is closed under these operations. Starting from an arbi- 
trary NDTA, it is possible to extend it to a Boolean-complete one ${ }^{2}$. If $\boldsymbol{r}_{\mathbf{1}}, \boldsymbol{r}_{\mathbf{2}}$ are two nodes, we write $r_{1} \vee r_{2}$ (resp. $r_{1} \wedge r_{2}, \neg r_{1}$ ) for some node $r$ such that $\mathcal{A} \llbracket r \rrbracket=\mathcal{A} \llbracket r_{1} \rrbracket \cup \mathcal{A} \llbracket r_{2} \rrbracket\left(\right.$ resp. $\left.\mathcal{A} \llbracket r_{1} \rrbracket \cap \mathcal{A} \llbracket r_{2} \rrbracket, \mathcal{V} \backslash \mathcal{A} \llbracket r_{1} \rrbracket\right)$.

The Canonicity property forces a canonical way to decompose the set $\mathcal{A} \llbracket r \rrbracket^{2}$ as a finite union of rectangles of the form $\mathcal{A} \llbracket r_{1} \rrbracket \times \mathcal{A} \llbracket r_{2} \rrbracket$. For instance, it disallows the following situation: $\left\{\left(r_{1}, r_{2}\right) \mid\left(r_{1}, r_{2}, r\right) \in \delta\right\}=\{(a, c),(b, c)\}$. In that case, the decomposition of $\mathcal{A} \llbracket r \rrbracket^{2}$ given by $\delta$ would have two rectangles with the same second component. To eliminate this situation, we can merge the two rectangles, to keep only $(a \vee b, c)$. We also want to avoid more complex situations, for instance where a rectangle in the decomposition of $\mathcal{A} \llbracket r \rrbracket^{2}$ is covered by the union of others rectangles in this decomposition. It is always possible to modify the transition relation $\boldsymbol{\delta}$ of a Boolean-complete NDTA to enforce the Canonicity property (first, by splitting the rectangles to enforce nonintersecting first-components, and then by merging rectangles with the same second component). This process does not break Boolean completeness since it doesn't change the class of languages defined by the automaton. The definition of Canonicity is asymmetric with respect to $\boldsymbol{r}_{1}$ and $\boldsymbol{r}_{2}$; this corresponds to the fixed traversal order of a tree during the run of a NUA (left-to-right).

We will use the word "type" to refer to the nodes of our fixed NDTA $\mathcal{A}$. Indeed, they correspond closely to the types of the CDuce (internal) type algebra, which support boolean operations and a canonical decomposition of products. Note that the set of types is finite, here. In what follows, we use $t, t_{1}, t_{2}$, $\ldots$ to range over nodes of the given NDTA, and $r, r_{1}, r_{2}, \ldots$ to range over nodes of the generated NUA. We also write $\llbracket t \rrbracket$ instead of $\mathcal{A} \llbracket t \rrbracket$. We define $\Delta^{2}(t)=\left\{\left(t_{1}, t_{2}\right) \mid\left(t_{1}, t_{2}, t\right) \in \delta\right\}$, and $\Delta^{0}(t)=\{a \mid(a, t) \in \delta\}$.

\subsection{Filters}

Even if we start with a single check to perform ("is the tree in $X$ ?"), several checks may have to be performed in parallel on a subtree ("is $\boldsymbol{v}_{\mathbf{1}}$ in $\boldsymbol{X}_{1}$ and/or in $\boldsymbol{X}_{\mathbf{3}}$ ?'); we will call any finite set of such checks a filter.

A filter is intended to be applied to any tree $v$ from a given language; for such a tree, the filter must compute which of its elements contain $v$.

Definition 5 Let $\boldsymbol{\tau}$ be a type. A $\tau$-filter is a set of types $\rho$ such that $\forall t \in$ $\rho . \llbracket t \rrbracket \subseteq \llbracket \tau \rrbracket$.

The result of a $\tau$-filter $\rho$ for a tree $v \in \llbracket \tau \rrbracket$, written $v / \rho$, is defined by:

$$
v / \rho=\{t \in \rho \mid v \in \llbracket t \rrbracket\}
$$

\footnotetext{
${ }^{2}$ The proof is outside the scope of this paper. In a nutshell, this can be done by adding nodes that represent formal boolean combinations of existing nodes (using a finite syntax for combinations, like disjunctive normal forms). See for instance [FCB02]. This process induces an exponential blowup of the size of the automaton, but this is not an issue in practice since we don't need to compute the whole automaton.
} 
DEFINITION 6 Let $\boldsymbol{\rho}$ be a $\tau$-filter. If $\boldsymbol{\rho}^{\prime} \subseteq \rho$, we write $\boldsymbol{\rho}^{\prime} \mid \boldsymbol{\rho}$ for the type:

$$
\tau \wedge \bigwedge_{t \in \rho^{\prime}} t \wedge \bigwedge_{t \in \rho \backslash \rho^{\prime}} \neg t
$$

(the $\tau$ in this formula is only useful for the case $\rho^{\prime}=\varnothing$ )

LEMma 7 Let $\boldsymbol{\rho}$ be a $\tau$-filter and $\boldsymbol{v}$ a tree in $\llbracket \tau \rrbracket$. Then $\boldsymbol{v} / \boldsymbol{\rho}$ is the only subset $\rho^{\prime} \subseteq \rho$ such that $v \in \llbracket \rho^{\prime} \mid \rho \rrbracket$.

Our construction consists of building a NUA whose states are pairs $(\tau, \rho)$ of a type $\tau$ and a $\tau$-filter $\rho$. Note that the set of all these pairs is finite, because we are working with a fixed NDTA to define all the types, so there is only a finite number of them.

\subsection{Discussion}

The type $\tau$ represents the static information we have about the tree, and $\rho$ represents the tests we want to perform on a tree $v$ which is known to be in $\tau$. The expected behavior of the automaton is:

$$
\forall v \in \llbracket \tau \rrbracket \cdot \mathcal{A}((\tau, \rho), v)=v / \rho
$$

Moreover, the state $(\tau, \rho)$ can simply reject any tree outside $\llbracket \tau \rrbracket$. Actually, we will build a NUA such that $\operatorname{Dom}((\tau, \rho))=\llbracket \tau \rrbracket$.

The rest of the section describes how the NUA should behave on a given input. It will thus mix the description of the expected behavior of the NUA at runtime and the (compile-time) construction we deduce from this behavior.

Results. In order to minimize the set of possible results for a state $(\tau, \rho)$, we consider only the $\rho^{\prime} \subseteq \rho$ that can be obtained for an input in $\tau$ :

$$
R((\tau, \rho))=\left\{\rho^{\prime} \subseteq \rho\left|\llbracket \rho^{\prime}\right| \rho \rrbracket \neq \varnothing\right\}
$$

Note that $\rho^{\prime}$ is in this set if and only if there is a $v \in \llbracket \tau \rrbracket$ such that $v / \rho=\rho^{\prime}$.

Left. Assume we are given a tree $v=\left(v_{1}, v_{2}\right)$ which is known to be in a type $\tau$. What can we say about $v_{1}$ ? Trivially, it is in one of the sets $\llbracket t_{1} \rrbracket$ for $\left(t_{1}, t_{2}\right) \in \Delta^{2}(\tau)$. We define:

$$
\pi_{1}(\tau)=\bigvee_{\left(t_{1}, t_{2}\right) \in \Delta^{2}(\tau)} t_{1}
$$

It is the best information we can find about $v_{1}$ (we use the assumption that the rectangles in the decomposition are not empty - this is part of the Canonicity property). Note that: $\llbracket \pi_{1}(\tau) \rrbracket=\left\{v_{1} \mid\left(v_{1}, v_{2}\right) \in \llbracket \tau \rrbracket\right\}$. 
Now assume we are given a $\tau$-filter $\rho$ that represents the tests we have to perform on $v$. Which tests do we have to perform on $v_{1}$ ? It is enough to consider those tests given by the $\pi_{1}(\tau)$-filter:

$$
\pi_{1}(\rho)=\left\{t_{1} \mid\left(t_{1}, t_{2}\right) \in \Delta^{2}(t), t \in \rho\right\}
$$

This set is indeed a $\pi_{1}(\tau)$-filter. It corresponds to our choice of performing any computation on $v_{1}$ which can potentially simplify the work we have to do later on $v_{2}$. Indeed, two different rectangles in $\Delta^{2}(t)$ for some $t \in \rho$ have different second projections because of the Canonicity property. This discussion suggests to take:

$$
\operatorname{left}((\tau, \rho))=\left(\pi_{1}(\tau), \pi_{1}(\rho)\right)
$$

Right. Let us continue our discussion with the tree $v=\left(v_{1}, v_{2}\right)$. The NUA performs some computation on $v_{1}$ from the state $\left(\tau_{1}, \rho_{1}\right)$ with $\tau_{1}=\pi_{1}(\tau)$ and $\rho_{1}=\pi_{1}(\rho)$. Let $\rho_{1}^{\prime}$ be the returned result, which is the set of all the types $t_{1} \in \rho_{1}$ such that $v_{1} \in \llbracket t_{1} \rrbracket$. What can be said about $v_{2}$ ? It is in the following type:

$$
\pi_{2}\left(\tau ; \rho_{1}^{\prime}\right)=\underset{\left(t_{1}, t_{2}\right) \in \Delta^{2}(\tau) \mid\left[t_{1} \wedge\left(\rho_{1}^{\prime} \mid \rho_{1}\right)\right] \neq \varnothing}{t_{2}}
$$

This type represents the best information we can get about $\boldsymbol{v}_{\mathbf{2}}$ knowing that $v \in \llbracket \tau \rrbracket$ and $v_{1} \in \llbracket \rho_{1}^{\prime} \mid \rho_{1} \rrbracket$. Indeed, its interpretation is:

$$
\left\{v_{2} \mid\left(v_{1}, v_{2}\right) \in \llbracket \tau \rrbracket, \rho_{1}^{\prime}=v_{1} / \rho_{1}\right\}
$$

Now we must compute the checks we have to perform on $v_{2}$. Let us consider a given type $t \in \rho$. If $\left(t_{1}, t_{2}\right) \in \Delta^{2}(t)$, we have $t_{1} \in \rho_{1}$, so we know if $v_{1} \in \llbracket t_{1} \rrbracket$ or not (namely, $v_{1} \in \llbracket t_{1} \rrbracket \Longleftrightarrow t_{1} \in \rho_{1}^{\prime}$ ). There is at most one pair $\left(t_{1}, t_{2}\right) \in \Delta^{2}(t)$ such that $v_{1} \in \llbracket t_{1} \rrbracket$. Indeed, two rectangles in the decomposition $\Delta^{\mathbf{2}}(t)$ have non-intersecting first projection (Canonicity). If there is such a pair, we must check if $\boldsymbol{v}_{\mathbf{2}}$ is in $\llbracket t_{\mathbf{2}} \rrbracket$ or not, and this will be enough to decide if $v$ is in $\llbracket t \rrbracket$ or not. We thus take:

$$
\pi_{2}\left(\rho ; \rho_{1}^{\prime}\right)=\left\{t_{2} \mid\left(t_{1}, t_{2}\right) \in \Delta^{2}(t), t \in \rho, t_{1} \in \rho_{1}^{\prime}\right\}
$$

This set has at most as many elements as $\rho$ by the remark above. Finally, the "right" transition is:

$$
\operatorname{right}\left((\tau, \rho), \rho_{1}^{\prime}\right)=\left(\pi_{2}\left(\tau ; \rho_{1}^{\prime}\right), \pi_{2}\left(\rho ; \rho_{1}^{\prime}\right)\right)
$$

Computing the result. We write $\tau_{2}=\pi_{2}\left(\tau ; \rho_{1}^{\prime}\right)$ and $\rho_{2}=\pi_{2}\left(\rho ; \rho_{1}^{\prime}\right)$. We can run the NUA from this state $\left(\tau_{2}, \rho_{\mathbf{2}}\right)$ on the tree $\boldsymbol{v}_{\mathbf{2}}$, and get a result $\rho_{\mathbf{2}}^{\prime} \subseteq \rho_{\mathbf{2}}$ collecting the $t_{2} \in \rho_{2}$ such that $v_{2} \in \llbracket t_{2} \rrbracket$. For a type $t \in \rho$, and a rectangle $\left(t_{1}, t_{2}\right)$ in its decomposition $\Delta^{2}(t)$, we have:

$$
v \in \llbracket t_{1} \rrbracket \times \llbracket t_{2} \rrbracket \Longleftrightarrow\left(t_{1} \in \rho_{1}^{\prime}\right) \wedge\left(t_{2} \in \rho_{2}^{\prime}\right)
$$


So the result of running the NUA from the state $(\tau, \rho)$ on the tree $v$ is:

$$
\delta^{2}\left((\tau, \rho), \rho_{1}^{\prime}, \rho_{2}^{\prime}\right)=\left\{t \in \rho \mid \Delta^{2}(t) \cap\left(\rho_{1}^{\prime} \times \rho_{2}^{\prime}\right) \neq \varnothing\right\}
$$

Result for symbols. Finally, we must consider the case when the tree $v$ is a symbol $a \in \Sigma$. The NUA has only to accept for the state $(\tau, \rho)$ trees in the set $\llbracket \tau \rrbracket$; so if $a \notin \Delta^{0}(\tau)$, we can let $\delta^{0}((\tau, \rho), a)$ undefined. Otherwise, we take:

$$
\delta^{0}((\tau, \rho), a)=\left\{t \in \rho \mid a \in \Delta^{0}(t)\right\}
$$

\subsection{Formal construction, soundness}

We can summarize the above discussion by an abstract construction of the NUA:

- the set of states are the pairs $(\tau, \rho)$ where $\tau$ is a type and $\rho$ a $\tau$-filter;

- $R((\tau, \rho))=\left\{\rho^{\prime} \subseteq \rho\left|\llbracket \rho^{\prime}\right| \rho \rrbracket \neq \varnothing\right\}$

- $\operatorname{left}((\tau, \rho))=\left(\pi_{1}(\tau), \pi_{1}(\rho)\right)$ where: $\pi_{1}(\tau)=\bigvee\left\{t_{1} \mid\left(t_{1}, t_{2}\right) \in \Delta^{2}(\tau)\right\}$ and $\pi_{1}(\rho)=\left\{t_{1} \mid\left(t_{1}, t_{2}\right) \in \Delta^{2}(t), t \in \rho\right\}$

- $\operatorname{right}\left((\tau, \rho), \rho_{1}^{\prime}\right)=\left(\pi_{2}\left(\tau ; \rho_{1}^{\prime}\right), \pi_{2}\left(\rho ; \rho_{1}^{\prime}\right)\right)$ where:

$\rho_{1}=\pi_{1}(\rho)$,

$\pi_{2}\left(\tau ; \rho_{1}^{\prime}\right)=\bigvee\left\{t_{2} \mid\left(t_{1}, t_{2}\right) \in \Delta^{2}(\tau), \llbracket t_{1} \wedge\left(\rho_{1}^{\prime} \mid \rho_{1}\right) \rrbracket \neq \varnothing\right\}$ and $\pi_{2}\left(\rho ; \rho_{1}^{\prime}\right)=\left\{t_{2} \mid\left(t_{1}, t_{2}\right) \in \Delta^{2}(t), t \in \rho, t_{1} \in \rho_{1}^{\prime}\right\} ;$

- $\delta^{2}\left((\tau, \rho), \rho_{1}^{\prime}, \rho_{2}^{\prime}\right)=\left\{t \in \rho \mid \Delta^{2}(t) \cap\left(\rho_{1}^{\prime} \times \rho_{2}^{\prime}\right) \neq \varnothing\right\}$;

- $\delta^{0}((\tau, \rho), a)=\left\{t \in \rho \mid a \in \Delta^{0}(t)\right\}$ if $a \in \Delta^{0}(\tau)$ (undefined otherwise)

This equations give explicitly for each state $q$ the set of results $R(q)$ and the transition functions for this state. This opens the door to a lazy construction of the NUA from an initial state, so as to build only the part of the NUA that is effectively used in a run. The abstract presentation however has the advantage of simplicity (exactly as for the abstract subset construction for the determinization of automata).

The construction has a nice property: the efficiency of the constructed NUA (that is, the positions where it will ignore subtrees of an input) does not depend on the type $\tau$ and the types in $\rho$ (which are syntactic objects), but only on the languages denoted by these types. This is because of the Canonicity property. As a consequence, there is no need to "optimize" the types before running the algorithm.

The following theorem states that the constructed NUA computes what it is supposed to compute. 
THEOREM 8 The above construction is well defined and explicitly computable. The resulting NUA satisfies the following properties for any state $(\tau, \rho)$ :

- $\operatorname{Dom}((\tau, \rho))=\llbracket \tau \rrbracket$

- $\forall v \in \llbracket \tau \rrbracket \cdot \mathcal{A}((\tau, \rho), v)=v / \rho$

- $\forall \rho^{\prime} \in R((\tau, \rho)) . \exists v \cdot \mathcal{A}((\tau, \rho), v)=\rho^{\prime}$

The third point simply states that there are no "useless" result (a result is useless if it cannot be obtained for a value in the domain). The proof of the theorem is by induction on trees, and follows the lines of the discussion above.

\subsection{An example}

In this section, we give a very simple example of a NUA produced by our algorithm. We assume that $\boldsymbol{\Sigma}$ contains at least two symbols $\boldsymbol{a}, \boldsymbol{b}$ and possibly others. We consider a type $t_{\boldsymbol{a}}$ (resp. $t_{\boldsymbol{b}}$ ) which denotes all the trees with only $a$ leaves (resp. $b$ leaves). Our static information $\tau_{0}$ is $t_{a} \vee t_{b}$, and the filter we are interested in is $\rho_{0}=\left\{t_{a}, t_{b}\right\}$. Assuming proper choices for the NDTA that defines the types, the construction gives for the initial state $q_{0}=\left(\tau_{0}, \rho_{0}\right)$ :

- $R\left(q_{0}\right)=\left\{\left\{t_{a}\right\},\left\{t_{b}\right\}\right\}$

- $\operatorname{left}\left(q_{0}\right)=q_{0}$

- $\operatorname{right}\left(q_{0},\left\{t_{a}\right\}\right)=\left(t_{a},\left\{t_{a}\right\}\right) ; \operatorname{right}\left(q_{0},\left\{t_{b}\right\}\right)=\left(t_{b},\left\{t_{b}\right\}\right)$

- $\delta^{2}\left(q_{0},\left\{t_{a}\right\},\left\{t_{a}\right\}\right)=\left\{t_{a}\right\} ; \delta^{2}\left(q_{0},\left\{t_{b}\right\},\left\{t_{b}\right\}\right)=\left\{t_{b}\right\}$

- $\delta^{0}\left(q_{0}, a\right)=\left\{t_{a}\right\} ; \delta^{0}\left(q_{0}, b\right)=\left\{t_{b}\right\} ; \delta^{0}\left(q_{0}, c\right)$ undefined if $c \neq a, c \neq b$

There is no need to give the transition functions for the states $q_{a}=\left(t_{a},\left\{t_{a}\right\}\right)$ and $q_{b}=\left(t_{b},\left\{t_{b}\right\}\right)$ because they each have a single result $\left(R\left(q_{a}\right)=\left\{\left\{t_{a}\right\}\right\}\right.$ and $\left.R\left(q_{b}\right)=\left\{\left\{t_{b}\right\}\right\}\right)$, so the NUA will simply skip the corresponding subtrees. The behavior of the NUA is simple to understand: it goes directly to the leftmost leaf and returns immediately. In particular, it traverses a single path from the root to a leaf and ignores the rest of the tree.

\subsection{Implementation}

We rely a lot on the possibility of checking emptiness of a type $(\llbracket t \rrbracket=\varnothing)$. For instance, the definition of $R((\tau, \rho))$ requires to check a lot of types for emptyness. All the techniques developed for the implementation of XDuce and CDuce subtyping algorithms can be used to do it efficiently. In particular, because of caching, the total cost for all the calls to the emptiness checking procedure does not depend on the number of calls (there is a single exponential cost), so they are "cheap" and we can afford a lot of them. CDuce also 
demonstrates an efficient implementation of the "type algebra" with boolean combinations and canonical decomposition.

The number of states $(\tau, \rho)$ is finite, but it is huge. However, our construction proceeds in a top-down way: starting from a given state $(\tau, \rho)$, it defines its set of results and its transitions explicitly. Hence we are able to build the NUA "lazily" (either by computing all the reachable states, or by waiting to consume inputs - this is how the CDuce implementation works).

We haven't studied the theoretical complexity of our algorithm, but it is clearly at least as costly as the inclusion problem for regular tree languages. However, in practice, the algorithm works well. It has been successfully used to compile non-trivial CDuce programs.

Preliminary benchmarks [BCF03] suggest very good runtime performances, and we believe that our compilation strategy for pattern matching is the main reason for that.

\section{Acknowledgments}

I would like to express my best gratitude to Haruo Hosoya for his help to improve the presentation of the paper. The referees of PLANX 2004 and ICALP 2004 also suggested significant improvements to the presentation.

\section{References}

[BCF03] VéroniqueBenzaken, Giuseppe Castagna, and Alain Frisch. CDuce: an XMLcentric general-purpose language. In ICFP, 2003.

[BPSM98] Tim Bray, Jean Paoli, and C. M. Sperberg-McQueen. Extensible markup language (XML) 1.0. In W3CRecommendation, 1998.

[FCB02] Alain Frisch, Giuseppe Castagna, and Véronique Benzaken. Semantic subtyping. In LICS, 2002.

[GP03] Vladimir Gapeyev and Benjamin Pierce. Regular object types. In FOOL, 2003.

[Hos00] Haruo Hosoya. Regular expression types for XML. Ph.D thesis. The University of Tokyo, 2000.

[HP02] Haruo Hosoya and Benjamin Pierce. Regular expression pattern matching for XML. Journal of Functional Programming, 2002.

[Lev03] Michael Levin. Compiling regular patterns. In ICFP, 2003.

[Nev02] Frank Neven. Automata theory for XML researchers. In SIGMOD Record, 31(3), 2002., 2002.

[NS98] Andreas Neumann and Helmư Seidl. Locating matches of tree patterns in forests. In Foundations of Software Technology and Theoretical Computer Science, pages 134-145, 1998. Extended abstract available at http: / /www.

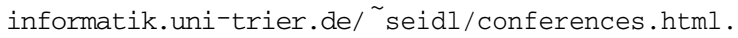

[SW03] Jerome Simeon and Philip Wadler. The essence of XML. In POPL, 2003.

[TBMM01] Henri S. Thompson, David Beech, Murray Maloney, and N. Mendelsohn. XML Schema part 1: Structures. In W3CRecommendation, 2001. 